\title{
ORIGINAL
}

\section{INCIDENCIA DE TUBERCULOSIS EN EL MUNICIPIO MARIANAO. CIUDAD DE LA HABANA, CUBA (1990-2000)}

\author{
Jorgelina D'fana Valdés (1), Shirley Cambell (2), Luisa Armas Pérez (1), Bárbaro Fraginal (2) y \\ Edilberto González Ochoa (1) \\ (1) Instituto de Medicina Tropical «Pedro Kouri». Ciudad de la Habana. Cuba \\ (2) Dirección Sectorial Municipal de Salud. Unidad de Análisis y Tendencias de Salud. calle: 116 ezq. 47. Marianao. \\ Ciudad la Habana.
}

\section{RESUMEN}

Fundamento: Para la eliminación de la tuberculosis es necesario vigilar su incidencia a nivel local, por ser éste el de acceso de los enfermos a los servicios de salud. El objetivo de este trabajo es describir la distribución y la tendencia de las tasas de incidencia, por áreas de salud y por grupos de edades, de la tuberculosis en el municipio Marianao, de Ciudad de la Habana, durante los periodos 1990-1994 y $1995-2000$.

Métodos: La serie temporal de las tasas de incidencia de 1990 1994 y 1995-2000 se analizó mediante una técnica de alisamiento exponencial con dos parámetros. Se estimó el porcentaje total y promedio anual de variación. Se hizo la estratificación de las áreas de salud.

Resultados: En 1990-1994 la tasa aumentó de 11,1 a 25,8 por 105 habitantes, (33\% incremento anual), siendo más notable en las áreas de los Policlínicos Finlay y Portuondo (incremento anual promedio de $112,7 \%$ y $36,2 \%$ respectivamente). Tomando en cuenta que en 1995 se realizó un refuerzo del programa de control, de 1995 a 2000 las tasas descendieron en 47,6\% en el municipio; las áreas de Finlay y Portuondo disminuyeron sus tasas en $79,9 \%$ y $39,7 \%$ respectivamente, mientras que en el área del Policlínico González Coro se incrementó un 58,3\% y el 27 de noviembre no mostró variación importante. El grupo de 60 años de edad y más fue en el que predominó el aumento, con un 49,8\% del total y sólo hubo un caso en menores de 15 años en el periodo. Durante el mismo, sólo se diag nosticaron seis casos de tuberculosis/Virus de le inmunodeficiencia humana. El área 27 de noviembre resultó la única ubicada en el nivel insatisfactorio, según la estratificación realizada.

Conclusiones: La tendencia de la tuberculosis en el municipio Marianao durante el periodo estudiado fue inicialmente ascendente y luego descendente. La enfermedad predominó en las personas adultas mayores.

Palabras claves: Tuberculosis. Incidencia. Tendencias. Estratificación. Enfermedades infecciosas y parasitarias.

\section{Correspondencia:}

Edilberto González Ochoa.

Instituto de Medicina Tropical «Pedro Kour

Apto 601 Marianao 13

Ciudad de la Habana

Cuba

Correo electrónico: ochoa@ipk.sld.cu.

\section{ABSTRACT}

Tuberculosis at the local level: Marianao Municipality, Havana City,Cuba (1990-2000)

Background: For the elimination of tuberculosis (TB) is necessary the surveillance of the incidence rate at the local level, as this is the first level of access to the health service. This study is aimed at providing a description of the spread and trend of the tuberculosis incidence rates in Marianao Municipality, Habana City by healthcare areas and age groups throughout the 1990-2000 period.

Methods: The time series for the 1990-1994 and 1995-2000 incidence rates were analyzed employing a two parameter exponential smoothing method. The total percentage and annual average of variation was estimated. The healthcare areas were stratified.

Results: In 1990 - 1994, the rate rose from 11.1 to 25.8 per 105 inhabitants (33\% of increase by year), having been most noticeable in Carlos J. Finlay and Portuondo heath areas (respectively annual average $112.7 \%$ y $36.2 \%$ increases). Taking into account that the control program was strengthened in 1995 , the rates dropped by $47.6 \%$ in this municipality during the $1995-2000$ period. The Finlay and Portuondo areas respectively lowered their rates by $79.9 \%$ y $39.7 \%$ while the Gonzalez Coro area showed a $58.3 \%$ rise and 27 de Noviembre area had no major change. The age and above 60 group had the higher percentage of cases, recording $49,8 \%$ of the total and only one case among children under age 15 for the period. Durin this same period, only six cases of tuberculosis/human inmunodeficiency virus were diagnosed. The 27 de Noviembre area was the only one found to be at the unsatisfactory level according to the stratification

Conclusions: An initially upward trend, followed by a downward trend in tuberculosis was found to exist in the Marianao Municipality throughout the period studied. This disease was predominant among older adults.

Key words: Communicable diseases. Tuberculosis. Incidence. Trends. Stratification 


\section{INTRODUCCIÓN}

La tuberculosis (TB) constituye uno de los problemas más graves de salud publica ${ }^{1}$, ya que sigue siendo la enfermedad que más personas mata en el mundo. En 1993 fue declarada por la OMS emergencia sanitaria mundial, y tiene una incidencia anual estimada de 400 mil casos en el Continente Americano y más de 2 millones de muertes por año en el mundo, siendo la mitad de los enfermos mayores de 15 años de edad. Es la patología re-emergente más frecuente en las personas jóvenes y tiende a ser más agresiva en el grupo de 15 a 45 años $^{2}$. Se incluye dentro de las seis enfermedades infecciosas que causan el $90 \%$ de las muertes por infecciones, principalmente en personas jóvenes. Entre los factores desencadenantes de esta situación pueden mencionarse la pandemia del VIH, la mala situación socioeconómica de diversos países, el aumento de la marginalidad y las migraciones, el debilitamiento de los programas de control y el aumento de la resistencia a las drogas anti-tuberculosas ${ }^{3}$.

En Cuba, después del año 1959, la situación de la tuberculosis mejoró notablemente4. En 1970 se implantó, y luego se generalizó en todo el país, un programa integrado en todos los servicios de salud, basado en el diagnóstico bacteriológico y en el tratamiento ambulatorio controlado (Cuba, Ministerio de Salud Pública/OPS. Programa Nacional de Control de la Tuberculosis, 1970). A partir de este momento la notificación de casos comenzó a descender, desde una incidencia de 14,3 por $10^{5}$ habitantes en 1972 a 4,7 por $10^{5}$ habitantes en $1991^{5}$. Sin embargo, a partir de 1992 hubo un nuevo incremento que llegó a 14,9 por 105 habitantes en 1994. Para controlar esta nueva situación, a finales de 1993 se realizó una revisión del programa, con el fin de fortalecer las intervenciones sectoriales y extrasectoriales y la operación de cada uno de sus componentes. En 1995 se logró detener el incremento de casos, iniciándose la disminución en la incidencia a partir de $1996^{6}$.
La provincia Ciudad de la Habana incrementó su tasa de notificación de 7,9 por $10^{5}$ habitantes en 1990 a 12,8 por 105 habitantes en el 19997 . El municipio Marianao presentó un decremento de la incidencia de la TB en el decenio de los años ochenta, hasta 12,5 por $10^{5}$ habitantes en 1990 . Debido a la importancia que tiene la vigilancia a nivel local para el control y eliminación de la TB, por ser el primer nivel de acceso de los enfermos a los servicios de salud, el objetivo de este trabajo es describir la distribución y tendencia de las tasas de notificación de la TB en el municipio Marianao, por áreas de salud y grupos de edades, en los períodos de 19901994 y 1995-2000, como parte de un estudio de intervención dirigido a evaluar distintas alternativas de localización de casos de TB, en condiciones de baja prevalencia de la enfermedad en un país de baja renta.

\section{SUJETOS Y MÉTODOS}

Se llevó a cabo un estudio ecológico descriptivo de series temporales, de las tasas de incidencia de tuberculosis por años, grupos de edad y áreas de salud del municipio Marianao. Se tomó el numero de casos notificados de TB (todas las formas) de 1990 hasta el 2000, obtenidos del registro de notificaciones del Departamento de Estadísticas y las estimaciones de la población realizadas por la Unidad de Análisis y Tendencias en Salud (UATS), ambas de la Dirección Municipal de Salud. La base de datos y el análisis se realizaron en Excel 2000 y en EVIEW. El abordaje del análisis de las tendencias de las tasas, se hizo a partir de la simple observación de los valores8; se empleó la técnica de alisamiento exponencial con dos parámetros. Se calcularon los porcentajes de variación global de las tasas, que se obtiene como sigue: Xo - Xn / Xo; y la variación promedio anual: $\mathrm{Xo}-\mathrm{Xn} / \mathrm{n}-1$, sea $\mathrm{Xo}=$ tasa de notificación de casos o tasa de incidencia del año inicial y $\mathrm{Xn}=$ Tasa de incidencia del ultimo año, de cada etapa analizada. Las tasas por grupo de edad para todo el periodo se calcu- 
laron utilizando como denominador la población media del periodo.

Para los casos notificados de $1990^{\text {a }} 1994$ se definió como caso nuevo de TB aquel diagnosticado y notificado por primera vez, en el cual se demostró la presencia del Mycobacterium tuberculosis por cualquier método diagnóstico ${ }^{9}$. En el año 1994 se produjo un cambio en el criterio de notificación en el Programa Nacional de Control de la Tuberculosis (PNCT) incluyendo a los sujetos que tienen examen directo y cultivo de esputo negativo y poseen imagen radiográfica compatible con el diagnostico de $\mathrm{TB}^{10}$. A partir de 1995 se produjo una intensificación en las intervenciones preventivas para $\mathrm{TB}^{11}$. Por tal motivo, y a efectos de un análisis correcto de los datos, dividimos el período de estudio en dos etapas: 1990-1994 y 1995-2000.

La intensificación de las acciones del PNCT en su edición de 1994 consiste en:

- Aumento de la localización e identificación de personas con síntomas respiratorios prolongados (14 días o más) en los servicios de los médicos de la familia y consultas externas de hospitales, elevando el estándar de evaluación desde $0.6 \%$ hasta $1.0 \%$;

- Ampliación de la definición de casos de tuberculosis que deben ser notificados, incluidos aquellas con baciloscopia y cultivos negativos pero con imagen radiográfica compatible con el diagnóstico de tuberculosis pulmonar o extrapulmonar;

- Reforzamiento del proceso de control de calidad para el diagnostico clínico y radiográfico de los casos de tuberculosis con bacteriología negativa;

- Reintroducción del proceso de control de calidad para el diagnóstico clínico y radiológico de los casos de tuberculosis con bacteriología negativa;
- Introducción de los criterios estándar orientados por la OMS/UICTER para la valoración de los desenlaces del tratamiento;

- Inclusión de la tuberculosis en el sistema de notificación semanal, numéricocolectiva, del sistema de información directa-Alerta/Acción de las Unidades de Análisis y Tendencias en salud (UATS) y de la Dirección Nacional de Estadísticas del Ministerio de Salud Publica;

- Implantación de un nuevo sistema de registro y análisis con destino al PNCT;

- Implantación de un nuevo formulario o cuestionario estándar para la investigación de contactos de los casos de tuberculosis;

- Ampliación de la quimioprofilaxis con isoniazida a los contactos extradomiciliarios;

- Fortalecimiento de los grupos Provinciales de Control de TB. Reinicio de la impartición del Curso Nacional de Administración de Programas de Control de la Tuberculosis y extensión en cascada de la capacitación en las provincias;

- Sistematización del proceso de supervisiones provinciales y municipales, y de las evaluaciones trimestrales;

- Otorgamiento por el MINSAP de la máxima prioridad al PNCT dentro de los programas de enfermedades transmisibles.

La Incidencia de la TB sólo puede ser obtenida mediante investigaciones de cohortes diseñadas al efecto, y en determinadas circunstancias tal vez puede estimarse a partir del riesgo anual de infección; para los efectos de este trabajo utilizamos el término de tasa de incidencia como expresión de tasa notificación de casos de TB. 
Para el periodo1995-2000 se estimó la Meta Operativa Parcial Deslizante (MOPaD) que consiste en el valor menor de las medias geométricas calculadas para las tasas de incidencia de cada área de salud. A partir del valor MOPaD se construyeron estratos calculando la razón $=$ Tasa de notificación de casos de TB 2000 / valor MOPaD y conformando cinco estratos o niveles: $\mathrm{I}=$ $<1$; II= 1-1.9; III= 2-2,9; IV= 3-3,9 y V= $>4,12$.

El municipio Marianao, ubicado en el oeste de la provincia Ciudad de la Habana, ocupa el noveno lugar en extensión territorial, con $21,69 \mathrm{Km}^{2}$, con una densidad de población de $6.341,30$ habitantes por $\mathrm{Km} 2$; el total de población estimada del municipio es de 137.597 habitantes; de ellos se estiman en menores de 15 años: 27.934, de 15-24 años: 27.358, de 25-49 años: 55.612, de 50-59 años: 14.345 y 60 y más: 21.711 habitantes. Cuenta con cuatro áreas de salud, y con un policlínico en cada una: el Policlínico Ramon G. Coro con 19.620 habitantes, el Policlínico Carlos J. Finlay con 48.476 habitantes, el Policlínico 27 de Noviembre con 27.984 habitantes y el policlínico Carlos M Portuondo con 41.463 habitantes; dotados de un laboratorio clínico donde, entre otras investigaciones, se realizan exámenes directos de esputo, los cuales se envían al laboratorio provincial para el cultivo (la primera muestra) y para el control de la calidad. El municipio cuenta con 81 consultorios médicos que atienden una población entre 700 a 800 habitantes cada uno mediante un médico y una enfermera de la familia que realiza, dentro de sus funciones, la vigilancia pasiva y activa de la TB, que consiste en la detección entre las personas que consultan en el consultorio medico o entre las visitadas en su domicilio, de los sujetos sintomáticos respiratorios, definidos como aquéllos que tienen tos y expectoración prolongada (durante 2 semanas o más). Al enfermo de TB diagnosticado y notificado se le indica el test de Elisa para VIH, como pesquisa de VIH+; además se cuenta con un Programa de
Control de Infecciones de Transmisión Sexual (ITS) y sida, que tiene dentro de sus objetivos la búsqueda activa de personas seropositivas, a través de la realización del test de ELISA en grupos de riesgos, como son: los donantes de sangre, mujeres embarazadas, enfermos de ITS, los que acuden voluntariamente y los casos de Tuberculosis (MINSAP. Programa de Vigilancia y Control ITS/SIDA).

\section{RESULTADOS}

En el periodo 1990-1994 la tasa de incidencia aumentó de 11,1 en 1990 a 25,8 por $10^{5}$ habitantes en 1994 , con un incremento medio anual de $33,1 \%$, observándose una tendencia ligeramente ascendente. En el período de 1990 a 1994 todas las áreas de salud incrementaron la incidencia anual, siendo este incremento más notable en los Policlínicos CJ Finlay y Portuondo, con $112,7 \%$ y $36,2 \%$ respectivamente.

De 1995 al 2000 se produjo un descenso de $47,6 \%$ en el municipio $(9,5 \%$ promedio anual). En el año 1995 las tasas en las distintas áreas fluctuaban desde 9,8 hasta 30,6 por $10^{5}$ habitantes, mientras en el año 2000 fueron de 6,1 por 105 hasta 17,8 por $10^{5}$ habitantes.

El área del policlínico González Coro incrementó sus notificaciones, mientras que en el área del policlínico CJ Finlay disminuyó y en el policlínico 27 de Noviembre se mantuvo estacionaria (tabla 1 ).

La distribución anual de las tasas de incidencia por grupos de edades fue similar en todas las áreas de salud, con cifras mucho más altas en el grupo de 60 y más años, al que correspondió el 39,7\% de todos los casos del municipio. Le siguió el grupo de 25 a 49 años con el 36,2\% y el de 15 a 24 años con el 8,1\% (tabla 2). En los menores de 15 años sólo se notificó un caso en todo el período (año 1997).

Rev Esp Salud Pública 2003, Vol. 77, N. ${ }^{\circ} 2$ 
INCIDENCIA DE TUBERCULOSIS EN EL MUNICIPIO MARIANAO. CIUDAD DE LA HABANA, CUBA (1990-2000)

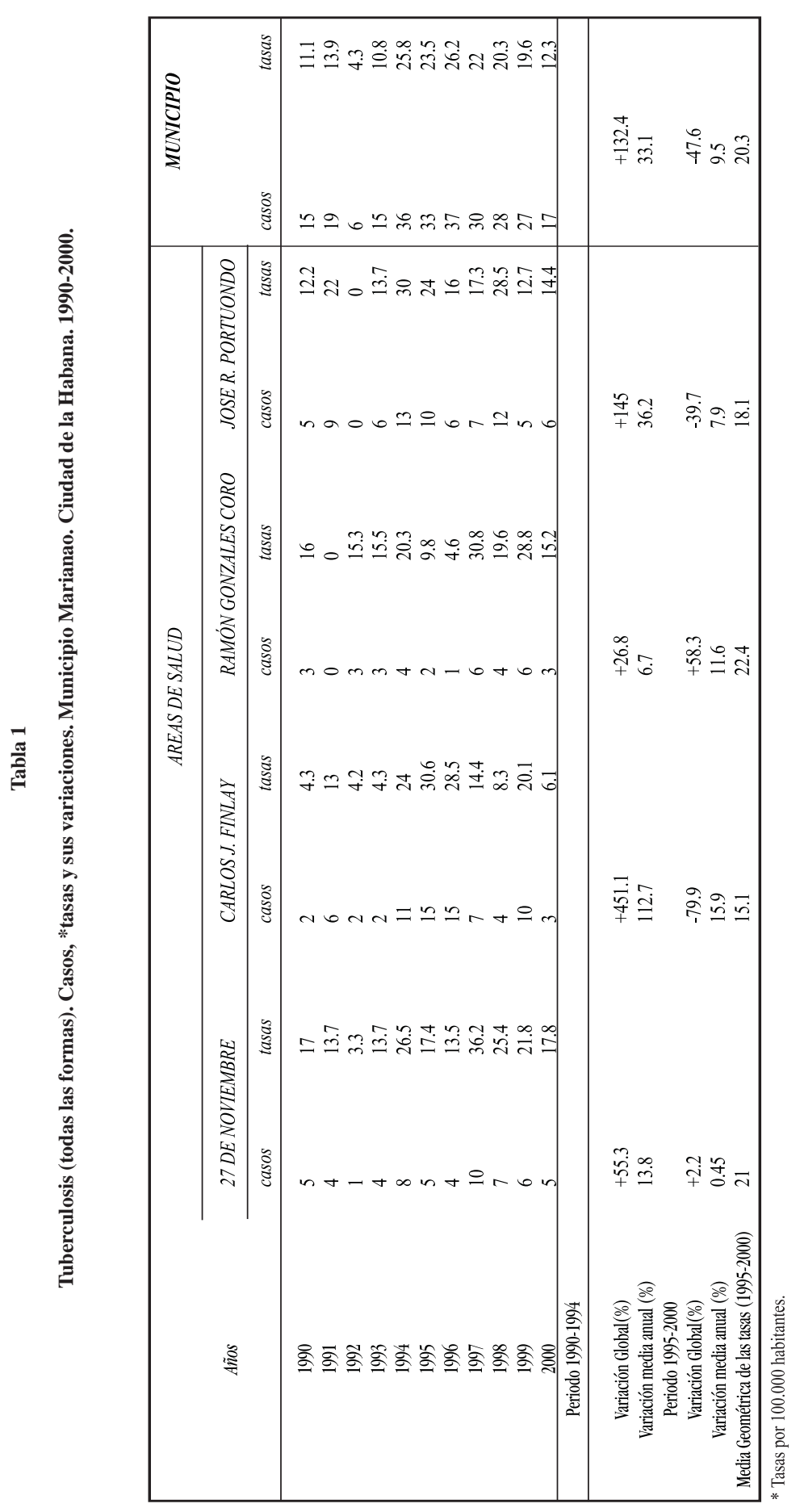

Rev Esp Salud Pública 2003, Vol. 77, N. ${ }^{\circ} 2$ 
Tabla 2

Distribución porcentual de los casos notificados de TB por grupos de edades en el municipio Marianao

\begin{tabular}{|c|c|c|c|c|c|c|c|c|c|}
\hline \multirow{2}{*}{ Años } & \multicolumn{2}{|c|}{ De 15 a 24} & \multicolumn{2}{|c|}{ De 25 a 49} & \multicolumn{2}{|c|}{ De 50 a 59} & \multicolumn{2}{|c|}{ Mas de 60} & \multirow{2}{*}{$\begin{array}{c}\text { TOTAL } \\
\text { casos }\end{array}$} \\
\hline & casos & $\%$ & casos & $\%$ & casos & $\%$ & casos & $\%$ & \\
\hline 1990 & 0 & 0 & 1 & 16.7 & 3 & 50 & 2 & 33.3 & 6 \\
\hline 1991 & 0 & 0 & 1 & 11.1 & 2 & 22.2 & 6 & 66.7 & 9 \\
\hline 1992 & 1 & 16.7 & 1 & 16.7 & 1 & 16.6 & 3 & 50 & 6 \\
\hline 1993 & 0 & 0 & 8 & 53.3 & 4 & 26.6 & 3 & 20 & 15 \\
\hline 1994 & 6 & 16.7 & 13 & 36.1 & 6 & 16.6 & 11 & 30.5 & 36 \\
\hline 1995 & 1 & 3 & 13 & 39.9 & 3 & 9.1 & 16 & 48.4 & 33 \\
\hline 1996 & 4 & 10.9 & 9 & 24.3 & 10 & 27 & 14 & 37.9 & 37 \\
\hline 1997 & 0 & 0 & 12 & 41.3 & 2 & 6.8 & 15 & 51.7 & 29 \\
\hline 1998 & 1 & 3.5 & 12 & 42.8 & 6 & 21.4 & 9 & 32.1 & 28 \\
\hline 1999 & 5 & 18.5 & 9 & 33.3 & 1 & 3.7 & 12 & 44.4 & 27 \\
\hline \multirow[t]{2}{*}{2000} & 2 & 11.8 & 9 & 53 & 1 & 5.9 & 5 & 29.4 & 17 \\
\hline & 20 & 8.1 & 88 & 36.2 & 39 & 15.5 & 96 & 39.3 & 243 \\
\hline
\end{tabular}

* No se conoce la edad de casos TB en los años 1990 y 1991 (9 y 10 respectivamente)

\section{Figura 1}

Tendencia de la tuberculosis en el municipio Marianao, Ciudad Habana. (1990-1995)

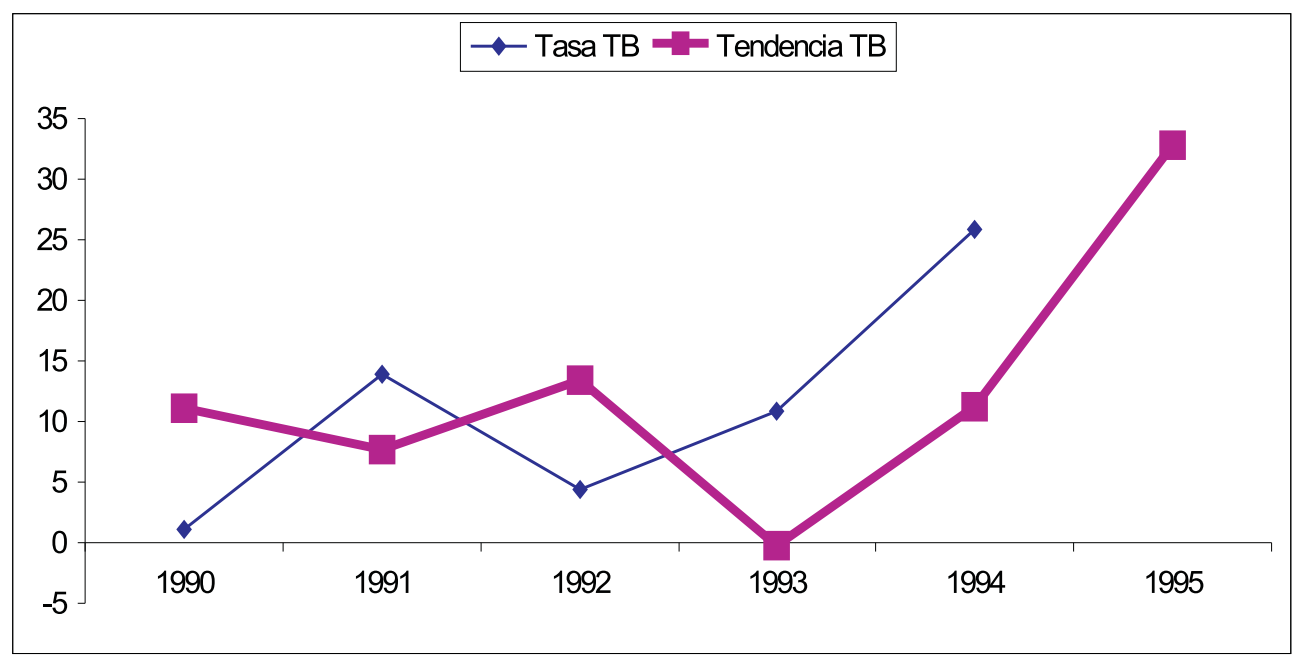

Durante la pesquisa de sujetos seropositivos al VIH se encontraron seis casos de coinfección TB/VIH, siendo el máximo en el año 1994, que se detectaron dos casos.
La conformación de estratos revela la existencia de dos niveles. En la MOPaD las áreas de los policlínicos Carlos J. Finlay y González Coro fueron ubicadas en el primer 
nivel y el 27 de Noviembre y José R. Portuondo en el segundo nivel. En la meta de San José solamente quedo el policlínico 27 de Noviembre en el segundo nivel (figura 1).

\section{DISCUSIÓN}

Se considera que el incremento de las tasas de incidencia de tuberculosis en el municipio Marianao corresponde, al igual que en el resto del país, a una combinación de factores como el recrudecimiento de la crisis económica, la disminución relativa de la prioridad de la tuberculosis en el conjunto de los problemas de salud del municipio, la reducción de algunos recursos necesarios, los cambios en la organización de las medidas de control, el deterioro de la vigilancia y de la conciencia sobre el riesgo de infección, y el debilitamiento general de la cantidad y la calidad de las acciones de localización de $\operatorname{casos}^{7}$. El hecho de que solo se notifiquen 6 casos de coexistencia de TB/SIDA acumulado en todo el periodo $(2,28 \%)$ inclina a pensar que la infección VIH no juega un papel importante en el incremento en el número de casos de la tuberculosis en ese periodo. No obstante, este aumento en el porcentaje de incremento de la incidencia en el municipio Marianao es menor que el observado en otros municipios del país.

Borroto y colaboradores reportaron un incremento de la incidencia de tuberculosis, en los municipios Playa $(+61,6)$, Plaza (+ $29,5)$, Centro Habana $(+34,7)$, Habana Vieja (+69) y Habana del Este $(+100,00)$, mientras que en otros descendió como en San Miguel del Padrón (-61,3), Arroyo Naranjo $(-51,7)$, Boyeros $(-40,0), 10$ de Octubre ($30,0)$ y La Lisa $(-29,2)^{7}$. La provincia Ciudad de la Habana incrementó el porcentaje de incidencia en $26,5 \%$ en el periodo de 1990 a 1994. En el país también se incrementó de 4,7 a 14,9 por $10^{5}$ habitantes de 1991 a 1994. En la ciudad de Nueva York la incidencia de TB es 4 veces el promedio nacional, mientras que en Harlem es 20 veces más alta ${ }^{13,14}$. En Haití, a pesar de que el $70 \%$ de los casos de TB no se notifican, se estima una incidencia anual superior a los 500 casos por 100.000 habitantes en las zonas urbanas y de 50 por 100.000 en las rurales ${ }^{15}$.

En 1993, la incidencia de la TB en la provincia Las Palmas, Gran Canaria, fue de

Tabla 3

Casos de TB, personas seropositivos al VIH y Coinfección TB-SIDA. Municipio Marianao, periodo 1990-2000

\begin{tabular}{|c|c|c|c|c|}
\hline Años & Casos TB & Casos Seropositivos al VIH & $\begin{array}{c}\text { Casos Confección TB- } \\
\text { /SIDA }\end{array}$ & $\begin{array}{c}\text { Proporción } \\
\text { co-infección/TB }\end{array}$ \\
\hline 1990 & 15 & 1 & 0 & 0 \\
\hline 1991 & 19 & 6 & 0 & 0 \\
\hline 1992 & 6 & 5 & 0 & 0 \\
\hline 1993 & 15 & 2 & 1 & 6.6 \\
\hline 1994 & 36 & 8 & 2 & 5.5 \\
\hline 1995 & 33 & 5 & 0 & 0 \\
\hline 1996 & 37 & 11 & 1 & 2.7 \\
\hline 1997 & 30 & 13 & 0 & 0 \\
\hline 1998 & 28 & 6 & 0 & 0 \\
\hline 1999 & 27 & 13 & 1 & 3.7 \\
\hline 2000 & 17 & 13 & 1 & 5.8 \\
\hline Total & 263 & 83 & 6 & 2.2 \\
\hline
\end{tabular}


Figura 2

Tendencia de la tuberculosis en el municipio Marianao, Ciudad Habana. (1995-2000)

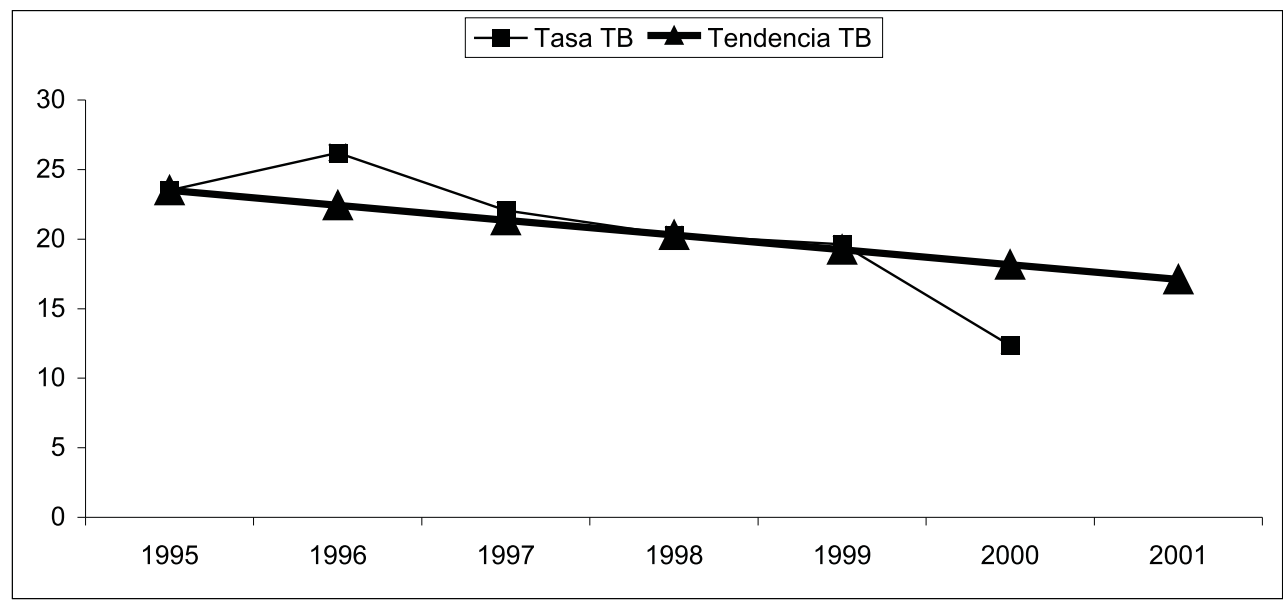

21,55 por $10^{5}$ habitantes (en los municipios: Mogán 4,98 y Veiza 39,02 x $10^{5}$ habitantes); la provincia de Santa Cruz de Tenerife (Gran Canaria) presentó también una evolución ascendente de 11,97 a 24,08 por 105 habitantes de 1985 a 1993 (en Tenerife Norte 8,37 y en Hierro 41,8 por $10^{5}$ habitantes) 16 . En el área de salud 15 de la Comunidad Valenciana en el periodo 1990-1993 la incidencia anual media fue de 34,81 por $10^{5}$ habitantes ${ }^{17}$.

Al parecer el fortalecimiento del PNCT a partir de 1995 provocó un nuevo descenso de las tasas de incidencia de la tuberculosis en el municipio Marianao. Éste presentó una incidencia anual por debajo de la provincia en el período de 1995-2000. La provincia de Ciudad de la Habana en el mismo período descendió en un $15,2 \%$, con una reducción media anual del 3\%. Los municipios considerados de mayor densidad poblacional, como Habana Vieja, Centro Habana y Cerro, se mantuvieron con cifras superiores a la media provincial durante el mismo período, con tasas de incidencia de 21,9, 22,5 y 15,3 por $10^{5}$ habitantes respectivamente; mientras los municipios periféricos, como Habana del este (9,9 por 105 habitantes), Lisa (10,3 por $10^{5}$ habitantes), Boyeros (12,2 por
105 habitantes) y Cotorro $\left(12,1\right.$ por $10^{5}$ habitantes) se mantuvieron con la menor incidencia durante el periodo ${ }^{7}$ y por debajo de las cifras de Marianao.

El predominio en los ancianos se corresponde con el del resto de la provincia y del país, y además coincide con lo reportado en los países desarrollados lo que, hasta cierto punto, habla a favor de las reactivaciones endógenas, como posible mecanismo causal de la enfermedad, pues la incidencia en jóvenes y niños se mantiene baja. No obstante se ha observado en el municipio un ligero incremento de los casos nuevos en el grupo de 15 a 24 años, como en otros muchos países en la actualidad ${ }^{2}$.

En la provincia Ciudad de La Habana al grupo de mayores de 60 años de edad correspondió el 36,1\% de los casos notificados en seis municipios (Habana Vieja, Cerro, Centro Habana, San Miguel del Padrón, Plaza y Boyeros) y la incidencia para este grupo de edad fue superior a la de la provincia, siendo la más baja en Regla con 4,1 por $10^{5}$ habitantes ${ }^{7}$.

La diferencia en las tasas observada en las áreas de salud tal vez podría explicarse con 
INCIDENCIA DE TUBERCULOSIS EN EL MUNICIPIO MARIANAO. CIUDAD DE LA HABANA, CUBA (1990-2000)

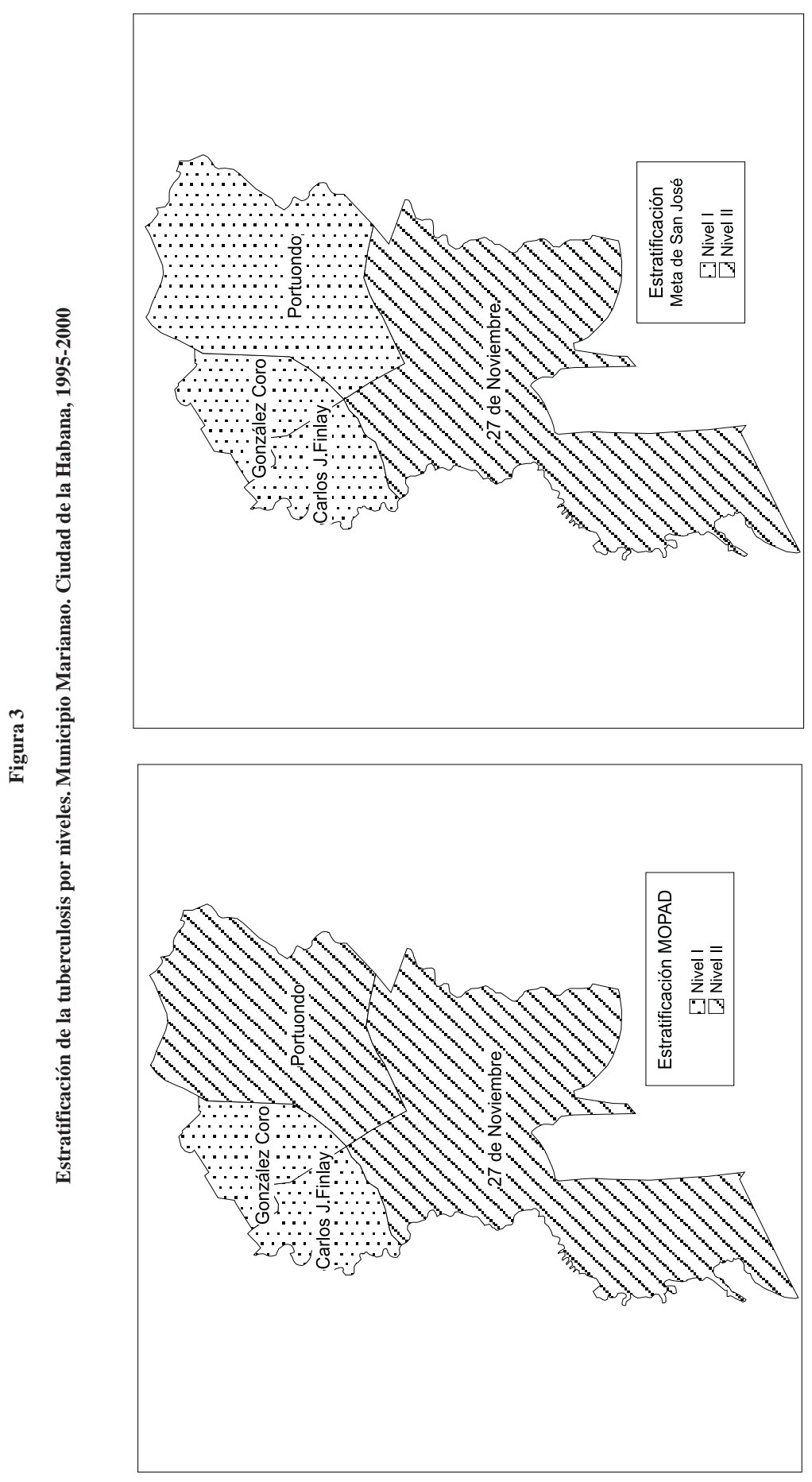

Rev Esp Salud Pública 2003, Vol. 77, NN. 2 
el mejor funcionamiento mantenido del programa en algunas de ellas ${ }^{18}$, pues las condiciones socioeconómicas y geográficas son muy semejantes. Los policlínicos Carlos $J$. Finlay y González Coro tienen distintas densidad poblacional (8.141,07 por Km2 y $23.998,01$ por $\mathrm{Km}^{2}$ ), la primera ha concentrado históricamente la mayor cantidad de casos notificados de TB en el periodo (Aplicación de una metódica para el análisis espacial y temporal de la incidencia de tuberculosis y su relación con factores socioeconómicas en un municipio de Cuba durante el periodo 1995-2000. Molina I, López C, Alonso R Instituto «Pedro Kouri»).

En conclusión, en Marianao, la Tuberculosis fue más frecuente en las áreas de salud C J Finlay y Portuondo en el periodo 19901994 y González Coro en el periodo 19952000. La enfermedad predomina en los adultos mayores, pero se observa un ligero incremento en los adultos jóvenes. La tendencia de las tasas de notificación de casos fue ascendente en la primera mitad del periodo estudiado, descendiendo en la segunda.

El descenso observado, en estos últimos años pone en evidencia un buen control de la situación y la recuperación paulatina del Programa Nacional de Control de la Tuberculosis.

\section{AGRADECIMIENTOS}

Los autores agradecen la colaboración prestada en la revisión y análisis de los datos a los compañeros Dra Susana Borroto, Lic. Alberto Baly y Lic. Ivette Molina, del Instituto de Medicina Tropical «Pedro Kouri».

\section{BIBLIOGRAFÍA}

1. Hashimoto T. The BCG vaccines for the prevention of tuberculosis in the world. Kekkaku 1997; 72(11):629-37.

2. OPS. Tuberculosis: la enfermedad re-emergente con mayor incidencia entre los jóvenes. Bol Oficina Sanit Panam 1999. FALTA INFORMACIÓN
3. World Health Organization. Tuberculosis 20002010: control, but not elimination. Communicable Diseases Control, Prevention \& Erradication. Ginebra: World Health Organization; 1999.

4. González E, Armas L, Baly A, Gálvez M, Ferrer G, Mesa A. Economic and social impact of the National Tuberculosis Control Program (NTCP) on the Cuban population. Cad Saúde Publica 2000; 16(3): 687-99.

5. González E, Armas L, Machín A. Tendencias por provincias de la Tuberculosis en Cuba. 1979 1993. Bol Oficina Sanit Panam 1995; 119(5):396404.

6. Marrero A, Caminero JA, Rodríguez R, Bitto NE. Towards elimination of tuberculosis in a low income country: the experience of Cuba, 1962-97. Tho$\operatorname{rax} 2000 ; 55$ : 39-45.

7. Borroto S, Armas L, González E, Peláez O, Servy J. Distribución y Tendencia de la Tuberculosis por grupos de edad y por municipios en Ciudad de la Habana. Rev Esp Salud Pública 2000; 74:507-15.

8. Camell F. Estadísticas Médicas y de Salud Pública 1985; 10(17):186

9. Ministerio de Salud Pública. Programa Nacional de Tuberculosis: manual de normas y procedimientos.. La Habana: MINSAP ECIMED;1999.p.1617.

10. Ministerio de Salud Pública. Actualización del Programa Nacional de Control de la Tuberculosis.. Dirección Nacional de Epidemiología, La Habana: Ministerio de Salud Pública; 1995.p. 3-5.

11. Ministerio de Salud Pública. Programa Nacional de Tuberculosis: manual de normas y procedimientos.. La Habana: MINSAP; 1999.p.16-17.

12. González E, Servy J, Armas L, Peláez O, Borroto S, Peralta M, Sánchez L, Gala A. Procedimientos alternativos para aplicar la estratificación epidemiológica de la Tuberculosis con indicador único. Boletín Epidemiológico IPK 2001. 11 (4): 185187.

13. Centers for Diseases Control. Morbidity for Tuberculosis in USA. MMWR 1999; 48 (33): 732-5.

14. Menzies D. Tuberculosis in big cities. The urban factor in tuberculosis. Conference on global health and the 1996 annual meeting of the IUATLD. Paris, France; 2-5 october 1996. Tuberc Lung Dis 1996; 77 (suppl 2): 16 . 
15. Hypolite PR, Pope JN. Infección por VIH y Tuberculosis en Haití. Bol Oficina Sanit Panam 1994; 118 (2):161-9.

16. Servicio Canario de salud. Situación de salud: morbilidad: Tuberculosis. Serie Plan de salud- 1 Docu- mento 2: www.gobcan.es/psc/pscO2/pscO2 2g. htm.

17. Ferrer E, Ballester D, Pérez F, Igual S, Fluixa R, Fullana C. Incidencia de la Tuberculosis pulmonar: aplicación del método de captura-recaptura. Gac Sanit 1997;11(3):115-121 